University of Nebraska - Lincoln

DigitalCommons@University of Nebraska - Lincoln

$1-2008$

\title{
SETTING UBVRI PHOTOMETRIC ZERO-POINTS USING SLOAN DIGITAL SKY SURVEY ugriz MAGNITUDES
}

Taylor S. Chonis

University of Nebraska-Lincoln, tschonis@bigred.unl.edu

C. Martin Gaskell

University of Nebraska-Lincoln, mgaskell@ucsc.edu

Follow this and additional works at: https://digitalcommons.unl.edu/physicsgaskell

Part of the Physics Commons

Chonis, Taylor S. and Gaskell, C. Martin, "SETTING UBVRI PHOTOMETRIC ZERO-POINTS USING SLOAN DIGITAL SKY SURVEY ugriz MAGNITUDES" (2008). Martin Gaskell Publications. 29.

https://digitalcommons.unl.edu/physicsgaskell/29

This Article is brought to you for free and open access by the Research Papers in Physics and Astronomy at DigitalCommons@University of Nebraska - Lincoln. It has been accepted for inclusion in Martin Gaskell Publications by an authorized administrator of DigitalCommons@University of Nebraska - Lincoln. 


\title{
SETTING UBVRI PHOTOMETRIC ZERO-POINTS USING SLOAN DIGITAL SKY SURVEY ugriz MAGNITUDES
}

TAYlor S. ChOnis And C. Martin GaSkell ${ }^{1}$

Department of Physics \& Astronomy, University of Nebraska, Lincoln, NE 68588-0111, USA; tschonis@ bigred.unl.edu, gaskell@astro.as.utexas.edu Received 2007 March 2; accepted 2007 October 29; published 2007 December 12

\begin{abstract}
We discuss the use of Sloan Digital Sky Survey (SDSS) ugriz point-spread function (PSF) photometry for setting the zero points of UBVRI CCD images. From a comparison with the Landolt (1992, AJ, 104, 340) standards and our own photometry we find that there is a fairly abrupt change in $B, V, R$, and $I$ zero points around $g, r, i \sim 14.5$, and in the $U$ zero point at $u \sim 16$. These changes correspond to where there is significant interpolation due to saturation in the SDSS PSF fluxes. There also seems to be another, much smaller systematic effect for stars with $g, r \gtrsim 19.5$. The latter effect is consistent with a small Malmquist bias. Because of the difficulties with PSF fluxes of brighter stars, we recommend that comparisons of ugriz and $U B V R I$ photometry should only be made for unsaturated stars with $g, r$, and $i$ in the range 14.5-19.5, and $u$ in the range 16-19.5. We give a prescription for setting the $U B V R I$ zero points for CCD images, and general equations for transforming from ugriz to UBVRI.
\end{abstract}

Key words: catalogs - standards - stars: fundamental parameters - surveys - techniques: photometric

\section{INTRODUCTION}

When CCD images of a field are taken it is necessary to determine the photometric zero points from stars of known magnitudes. It is, however, not unusual for there to be no stars with $U B V R I$ photometry available. Fortunately, the Sloan Digital Sky Survey (SDSS) now provides homogenous ugriz photometry for stars in a large fraction of the northern sky out of the plane of the Milky Way. Technical details of the SDSS are given in York et al. (2000) and Stoughton et al. (2002). The ugriz system (Fukugita et al. 1996) is significantly different from the widely used UBVRI Johnson-Cousins system (Cousins 1976), so it is necessary to transform between the two systems. A number of papers (Fukugita et al. 1996; Smith et al. 2002; Karaali et al. 2003, 2005; Bilir et al. 2005; Jordi et al. 2006; Rodgers et al. 2006; Ivezic et al. 2007; Davenport et al. 2007; Bilir et al. 2008) have considered the transformations between ugriz and UBVRI (see Section 6 for a discussion of these transformations).

During the course of using SDSS ugriz photometry to establish the zero points for comparison stars for photometry of active galactic nuclei (AGN), we noticed that the zero points were different for the fainter stars in a field than for the brighter stars. The difference was in the sense that stars with $g \lesssim 14$ were systematically brighter than predicted from the SDSS magnitudes. The difference did not seem to depend on the color of the stars and a check of the CCD used showed no evidence for nonlinearity. A subsequent comparison of magnitudes of Landolt standards (Landolt 1992) revealed a similar zero-point difference for stars brighter or fainter than $r \sim 14$.

In this paper we report the results of our investigation of the limitations of using SDSS photometry for bright stars, and give a prescription for setting zero points in CCD images taken through $U B V R I$ filters.

\section{TRANSFORMATION EQUATIONS}

We obtained ugriz magnitudes from SDSS data release 5 $(\mathrm{DR} 5)^{2}$ (Abazajian et al. 2005) for the Landolt (1992) standard stars in SDSS fields. We first removed very blue and red stars

\footnotetext{
1 Present Address: Department of Astronomy, University of Texas, Austin, TX 78712-0259, USA.

2 See http://www.sdss.org/dr5/.
}

outside the ranges $0.08<(r-i)<0.5$ and $0.2<(g-r)<1.4$. We then plotted the $(r-i)$ versus $(g-r)$ color-color diagram and removed outlying points more than 2.5 standard deviations from the linear least-squares fit. We derived transformation equations only for stars with $r>14$. A few points lying more than 2.5 standard deviations away from the least-squares fits were removed. We obtained the following transformations:

$$
\begin{aligned}
& B=g+(0.327 \pm 0.047)(g-r)+(0.216 \pm .027) \\
& V=g-(0.587 \pm 0.022)(g-r)-(0.011 \pm .013) \\
& R=r-(0.272 \pm 0.092)(r-i)-(0.159 \pm .022) \\
& I=i-(0.337 \pm 0.191)(r-i)-(0.370 \pm .041)
\end{aligned}
$$

As is well known, transformations to $U$ are particularly problematic. Since our aim is only to give a prescription for setting $U B V R I$ zero points rather than to obtain transformations valid for individual stars for astrophysical purposes, we determined the transformation for the $U$ filter as follows. First we removed all stars that were more than 2.5 standard deviations from a linear fit in four-dimensional $(u-g),(g-r),(r-i),(i-z)$ color space. For the remaining stars with no saturation warning flags, we restricted ourselves to stars with $1<(u-g)<2$ and $u>16$. For these stars we found no statistically significant dependence on the $(u-g)$ color. This is not surprising since, of the SDSS ugriz filters, the passband of the $u$ filter agrees most closely with the Johnson-Cousins passbands. The transformation for $U$ is thus

$$
U=u-0.854 \pm 0.007
$$

The standard errors in the zero points given by equations (1)(5) for $U, B, V, R$, and $I$ are $\pm 0.007,0.007,0.005,0.005$, and 0.009 respectively.

\section{MAGNITUDE DEPENDENCES}

In Figures 1-5 we show the dependences of the differences between the UBVRI magnitudes observed by Landolt (1992) and those calculated using Equations (1)-(5) versus $u, g, r$, or 


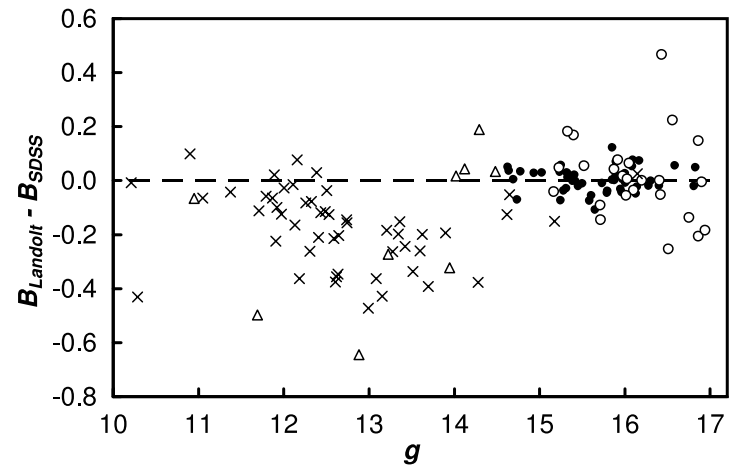

Figure 1. Residuals of observed Landolt $B$ magnitudes minus $B$ predicted from SDSS ugriz photometry versus $g$. The filled circles and crosses are stars from Landolt (1992). Stars with SDSS saturation warning flags are shown as crosses. The open circles and triangles are from our CCD photometry with a $0.4 \mathrm{~m}$ telescope. Stars with saturation warning flags are shown as triangles. The horizontal dashed line shows the zero point determined from the fainter stars.

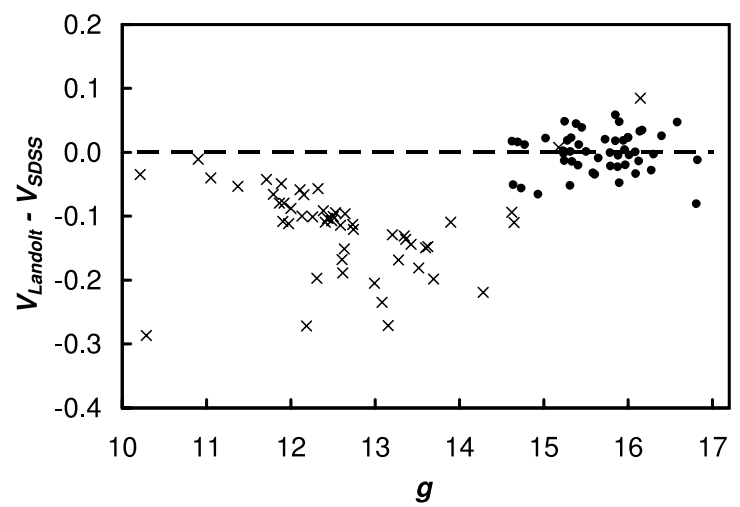

Figure 2. Residuals of observed Landolt $V$ magnitudes minus $V$ predicted from SDSS ugriz photometry plotted against $g$. Symbols are as in Figure 1 . The horizontal dashed line shows the zero point determined from the fainter stars.

$i$ color. ${ }^{3}$ It can be seen that in each case the SDSS magnitudes underpredict the $B V R I$ magnitudes by $\sim 0.15 \mathrm{mag}$ for the brighter stars and the $U$ magnitude by up to $\sim 2$ mag. In Figure 1 we also show the residuals in $B$ for stars for which we obtained $B$-band photometry as part of our AGN monitoring program. The systematic differences we see for these stars are consistent with those found from the Landolt (1992) standards. Since our CCD photometry was obtained with a completely different setup from the Landolt (1992) photomultiplier photometry, the agreement removes the possibility that the magnitude dependence is due to a hitherto undetected systematic effect in the Landolt (1992) photometry. The effect must arise instead from the calculation of PSF magnitudes in the SDSS data reduction pipeline when there is saturation of bright stars. In Figures 1-5 we have indicated with crosses and triangles which stars have saturation warning flags in the SDSS data base. Clearly, any star with a saturation warning flag associated with it should not be used for determining photometric zero points. In addition, extreme caution should be used when using photometry of these saturated stars in any application. It is interesting that apart from the abrupt change at $g, r, i \sim 14$ the PSF magnitudes are surprisingly good up to $g, r, i \sim 11$.

\footnotetext{
${ }^{3}$ Since we have restricted ourselves to stars with a fairly narrow range of color falling near a linear $(r-i)$ versus $(g-r)$ relationship, Figures 1-5 look similar if a different ugriz filter is plotted on the horizontal axis.
}

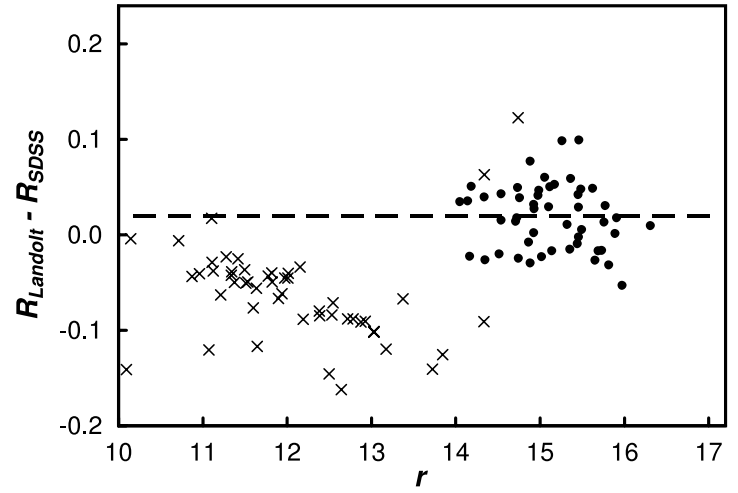

Figure 3. Residuals of observed Landolt $R$ magnitudes minus $R$ magnitudes predicted from SDSS ugriz photometry plotted against $r$. Symbols are as in the previous figures. The horizontal dashed line shows the zero point determined from the fainter stars

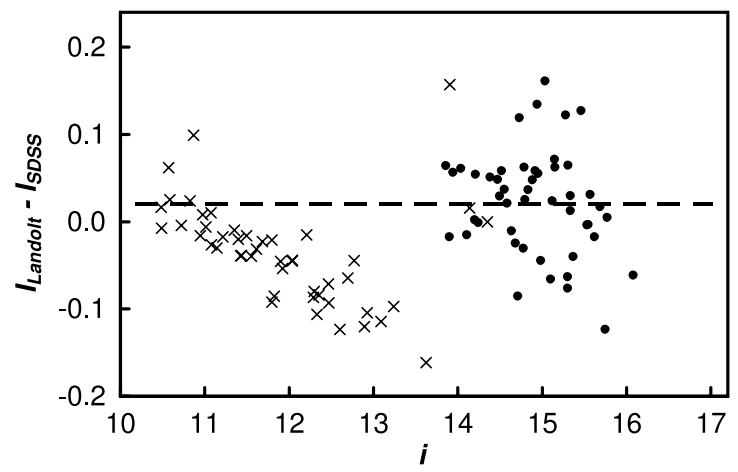

Figure 4. Residuals of observed Landolt $I$ magnitudes minus $I$ magnitudes predicted from SDSS ugriz photometry versus $i$. Symbols are as in the previous figures. The horizontal dashed line shows the zero point determined from the fainter stars.

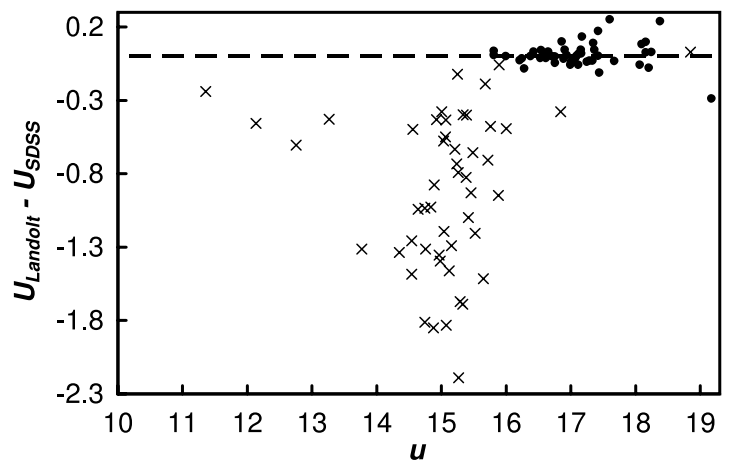

Figure 5. Residuals of observed Landolt $U$ magnitudes minus $U$ predicted from SDSS $u$ photometry versus $u$. Symbols are as in the previous figures. The horizontal dashed line shows the zero point determined from the fainter stars. Note that the vertical scale is larger than in the previous figures because of the larger standard errors associated with the $u$ magnitude saturation.

\section{FAINT STARS}

Although our main concern in this paper has been to investigate SDSS photometry of bright stars, we also looked for systematic effects at faint levels. Jordi et al. (2006) have derived transformations between griz and BVRI photometry for a large number of stars (see Jordi et al. (2006) for a description of the data sources). Their data set is inhomogeneous but includes a number of faint stars $(V>20)$. The Jordi et al. (2006) data show a large scatter (see figures in their paper), so in 


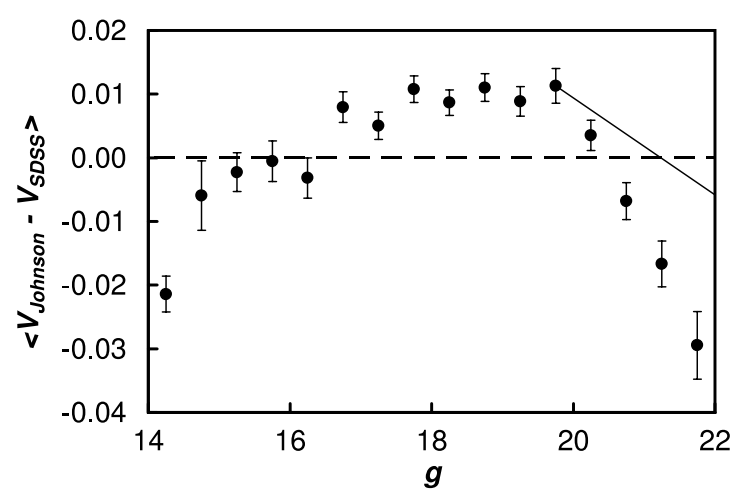

Figure 6. Mean residuals of observed $V$ magnitudes minus $V$ magnitudes predicted from SDSS ugriz photometry shown as a function of $g$ for the large photometric sample compiled by Jordi et al. (2006). The error bars show the standard errors in the means. Note that the vertical axis scale is magnified ten times compared with the scales in Figures 1-4. The horizontal dashed line shows the expected relationship if there are no magnitude-dependent effects. The thin solid line shows the expected effect of a Malmquist bias (see the text).

Figure 6 we show mean $V_{\text {Johnson }}-V_{\text {SDSS }}$ residuals as a function of $g$ for these data. For $14.5<g<17.5$ the mean residuals are, on average, close to zero (the horizontal line). Jordi et al. (2006) exclude stars with $r<14$ because of concerns with saturation effects, but it can be seen that the average residuals show a systematic deviation in the brightest bin $(g=14.25)$ in the same sense as we find in Figure 2 (but note that the scale in Figure 6 has been magnified by a factor of 10). There is also a systematic deviation for $g>19.5$. This systematic deviation is smaller, however, than the effect we find at $g \sim 14$.

One factor in the turndown for $g>19.5$ in Figure 6 could be Malmquist bias. ${ }^{4}$ Comparing the quoted standard errors for the photometry in the data set used by Jordi et al. (2006) with the errors in the $g$-band SDSS photometry shows that the SDSS photometric standard errors are several times larger than the standard errors in the other photometry at faint magnitudes. We simulated the effects of Malmquist bias with Monte Carlo simulations by creating artificial $B V R I$ photometry of 1,000 faint SDSS stars whose magnitudes had Gaussian noise added to them that was proportional to the quoted SDSS standard errors. The thin solid line in Figure 5 shows the effect of the bias. This should be regarded as a lower limit to the Malmquist bias. The slope of the Malmquist bias will increase with increasing random differences between the SDSS magnitudes and the $B V R I$ photometry, and could easily be twice as great as shown. In addition to the effects of Malmquist bias, there could be small systematic differences in the transformations and zero points for the inhomogeneous data sets used by Jordi et al. Different populations of stars at faint magnitudes could also be a factor. These other uncertainties could be the cause of the very slight systematic effect over the intermediate range $15<g<20$.

\section{DETERMINING ZERO POINTS IN CCD IMAGES}

We offer the following prescription for determining the zero points of CCD images taken through standard UBVRI filters. After instrumental magnitudes have been determined for all stars in the field, the stars are matched up with stars returned by the SDSS Skyserver. ${ }^{5}$ A $(g-r)$ versus $(r-i)$ color-color plot should be made for all stars without saturation warning flags. The list

\footnotetext{
${ }^{4}$ We are grateful to the referee for suggesting this possibility.

${ }^{5}$ http://cas.sdss.org/astro/en/tools/search/radial.asp.
}

should then be cleaned to remove all stars whose colors lie outside the ranges given in Section 2, and which lie far from the linear color-color relationship. The needed UBVRI magnitudes are then found using Equations (1)-(5) and the mean photometric zero points set using the average from the fainter stars.

\section{DISCUSSION}

The transformations we give in Equations (1)-(5) are consistent with the range of previously published transformations. Because our linear transformation equations are derived for a practical purpose of calibrating UBVRI photometry, and are available for each of the Johnson-Cousins filters individually, our transformations are different in nature from those previously published. We briefly summarize here previously published transformations and discuss how they differ from the ones given above. Fukugita et al. (1996) give synthetic transformations from $U B V R I$ to $u^{\prime} g^{\prime} r^{\prime} i^{\prime} z^{\prime}$. Smith et al. (2002) gave transformations between $U B V R I$ and $u^{\prime} g^{\prime} r^{\prime} i^{\prime} z^{\prime}$ magnitudes observed with the Photometric Telescope (PT) at Apache Point Observatory for some filters and for colors. Rodgers et al. (2006) give improved color transformations between $u^{\prime} g^{\prime} r^{\prime} i^{\prime} z^{\prime}$ and $U B V R I$ for main-sequence stars. They also consider higher-order color terms. It is important to note the difference between $u^{\prime} g^{\prime} r^{\prime} i^{\prime} z^{\prime}$ and ugriz. This is discussed in Smith et al. (2007). Additional technical details concerning the difference between the two systems as well as transformations between them are discussed in Tucker et al. (2006). Jordi et al. (2006) give color transformations between ugriz as observed with the SDSS $2.5 \mathrm{~m}$ telescope (rather than the PT) and UBVRI. Additional transformations are given by Jester et al. (2005), Karaali et al. (2003, 2005), Bilir et al. (2005, 2008) and Davenport et al. (2007). Some of the transformations including Ivezic et al. (2007) consider polynomials in the color terms, but we found no need for higher-order terms for the restricted range of colors we consider. Note that the above cited transformations consider only colors, transform from $U B V R I$ to $u g r i z$, are derived for the $u^{\prime} g^{\prime} r^{\prime} i^{\prime} z^{\prime}$ system, or give transformations only for select Johnson-Cousins filters.

In this paper, our aim has been to give a practical means of photometrically calibrating UBVRI CCD images. Researchers who are interested in astrophysical applications of SDSS photometry (such as the determination of spectroscopic parallaxes or fitting theoretical isochrones to HR diagrams) are referred to the above-mentioned papers because the ugriz to UBVRI transformations depend on the luminosity class and metallicity of the stars. We have minimized these effects for zero-point setting by using a fairly tight color selection.

We are grateful to Katrin Jordi for supplying the data from Jordi et al. (2006) in a machine-readable format, to Robert Lupton for useful discussion of the SDSS handling of PSF saturation, and the referee for useful comments. We wish to thank Tom Miller for making the photometric observations possible. This research has been supported by National Science Foundation grant through AST 03-07912 and the University of Nebraska UCARE program.

\section{REFERENCES}

Abazajian, K., et al. 2005, AJ, 129, 1755

Bilir, S., Karaali, S., \& Tunçel, S. 2005, Astron. Nachr., 326, 321

Bilir, S., Ak, S., Karaali, S., Cabrera-Lavers, A., Chonis, T. S., \& Gaskell, C. M. 2008, MNRAS, in press, (arXiv astro-ph/0711.4356)

Cousins, A. W. J. 1976, MmRAS, 81, 25 
Davenport, J. R. A., Bochanski, J. J., Covey, K. R., Hawley, S. L., West, A. A. \& Schneider, D. P. 2007, AJ, 134, 2430

Fukugita, M., Ichikawa, T., Gunn, J. E., Doi, M., Shimasaku, K., \& Schneider, D. P. 1996, AJ, 111, 1748

Ivezic, Z., et al. 2007, in in ASP Conf. Ser. 364, The Future of Photometric, Spectrophotometric, and Polarimetric Standardization, ed. C Sterken, 165 Jester, S., et al. 2005, AJ, 130, 873

Jordi, K., Grebel, E. K., \& Ammon, K. 2006, A\&A, 460, 339

Karaali, S., Karatas, Y., Bilir, S., Ak, S. G., \& Hamzaoglu, E. 2003, Publ. Astron. Soc. Aust., 20, 270
Karaali, S., Bilir, S., \& Tunçel, S. 2005, Publ. Astron. Soc. Aust., 22,24

Landolt, A. U. 1992, AJ, 104, 340

Rodgers, C. T., Canterna, R., Smith, J. A., Pierce, M. J., \& Tucker, D. L. 2006, AJ, 132, 989

Smith, J. A., et al. 2002, AJ, 123, 2121

Smith, J. A., et al. 2007, ASPC, 364, 91

Stoughton, C., et al. 2002, SPIE, 4836, 339

Tucker, D. L., et al. 2006, Astron. Nachr., 327, 821

York, D., et al. 2000, AJ, 120, 1579 